\title{
The Study of Treatment for Renal Insufficiency: Data and Evaluation (STRIDE), a National Registry of Chronic Kidney Disease
}

\author{
Madhumathi Rao, * Annamaria T. Kausz, * Don Mitchell, ${ }^{\dagger}$ Sari Heller Ratican, ${ }^{\dagger}$ \\ Francie Lin, ${ }^{\dagger}$ Sally Burrows-Hudson, ${ }^{\dagger}$ Fritz Port, ${ }^{\ddagger}$ and Brian J. G. Pereira ${ }^{\star}$ \\ *New England Health Care Foundation, Boston, Massachusetts, ${ }^{\dagger}$ Amgen Inc., Thousand Oaks, California, \\ and ${ }^{\ddagger}$ Renal Research Institute, University of Michigan, Ann Arbor, Michigan
}

\begin{abstract}
Optimization of care in patients with chronic kidney disease (CKD) could be the key to improved clinical and economic outcomes, both during the phase of CKD as well as in patients with end-stage renal disease (ESRD). CKD is a major public health problem that has been insufficiently studied. There is little published information on outcomes among CKD patients, specifically, data on mortality, morbidity, and quality of life. Indeed, recent efforts by the National Kidney Foundation (NKF) have served to define the classification, evaluation, and

approach to management of CKD in practice. The Study of Treatment for Renal Insufficiency: Data and Evaluation (STRIDE) registry is an initiative to study CKD patients in nephrology practices across the country. It is a prospective observational study whose objective is to profile demographic and clinical variables, practice patterns, comorbid conditions, quality of life, and outcomes in a nationally based sample of CKD patients. This article details the design, methodology, and process of enrollment into the registry.
\end{abstract}

There were 243,390 individuals on dialysis in the United States and another 101,000 Medicare kidney transplant patients at the end of 1999 (1). There is an average increase of about $7-9 \%$ in the yearly prevalence of end-stage renal disease (ESRD) (2). Despite improvements in the management of complications of kidney disease as well as in dialysis technologies, the morbidity and mortality associated with ESRD remain very high. Indeed, the gross annual mortality rates among dialysis patients are approximately $15-20 \%$, and 5- and 10-year survival rates are $38 \%$ and $21 \%$, respectively (2).

More recently, it has been recognized that factors associated with adverse outcomes among patients with ESRD, such as anemia, cardiac disease, hypertension, diabetes and malnutrition, exist for an extended period of time before the development of ESRD (3). It is also apparent that there are missed opportunities for improving the care of patients with chronic kidney disease (CKD) before the initiation of dialysis. Studies of incident dialysis patients in the mid-1990s indicated that more than half began ESRD therapy with hematocrits less than $28 \%$ and serum albumin less than $3.5 \mathrm{~g} / \mathrm{dl}$, and fewer than one-fourth received erythropoietin (EPO) $(1,4)$. Most patients with CKD first come under the care

Address correspondence to: Brian J. G. Pereira, MD, Division of Nephrology, New England Medical Center, Tufts University School of Medicine, 750 Washington St., Box 5224, Boston, MA 02111.

Seminars in Dialysis-Vol 15, No 5 (September-October) 2002 pp. 366-369 of a nephrologist only during the last few months prior to the initiation of dialysis (5). Late referral to a nephrologist has been associated with increased mortality and hospitalizations, suboptimal vascular access and anemia management, and inadequate control of metabolic parameters compared to patients with a longer duration of nephrology care (6-9). Optimization of care during earlier stages of CKD, well before the initiation of renal replacement therapy (RRT) for ESRD, should theoretically have a favorable impact on clinical outcomes $(9,10)$.

Estimates derived from the Third National Health and Nutrition Examination Survey (NHANES III) suggested that there were more than 6.2 million individuals with elevation of serum creatinine levels $\geq 1.5 \mathrm{mg} / \mathrm{dl}$ in the United States (11). More recently, estimates derived from a study of administrative data from a single health maintenance organization suggest that more than 9 million people in the United States have at least one elevated serum creatinine level (12). A reanalysis of the NHANES III data by the National Kidney Foundation Kidney Disease Outcomes Quality Initiative (K/DOQI) suggests that there are more than 20 million Americans with CKD, defined as a glomerular filtration rate (GFR) of less than $90 \mathrm{ml} / \mathrm{min} / 1.73 \mathrm{~m}^{2}$ or the presence of markers of kidney damage such as proteinuria (13). CKD represents a "silent epidemic," given that CKD does not become symptomatic until late in the disease progression, imposing a significant economic impact. Underrecognition and undertreatment of CKD may lead to suboptimal management of comorbid conditions and the institution of RRT late into the illness, inflating costs 
related to the increased morbidity and hospitalization in patients with CKD and ESRD (14).

There is little published information on outcomes among CKD patients; specifically, data on mortality, morbidity, and quality of life. The Study of Treatment for Renal Insufficiency: Data and Evaluation (STRIDE) registry was undertaken to examine practice patterns, comorbid conditions, quality of life, and outcomes among a nationally based sample of CKD patients. The study objective was to further the understanding of practices in the care of patients with CKD that are associated with improved patient outcomes.

\section{Registry Design}

STRIDE is a prospective observational cohort study of CKD patients. The study protocol was approved by a central institutional review board (IRB). Each enrolling physician-investigator obtained independent IRB approval as necessary and agreed to adhere to the principles of good clinical practice (15).

\section{Physician and Site Recruitment}

A national sample of 4600 sites was considered. Internal medicine, nephrology, and cardiology practices treating patients with CKD were included, with emphasis on practices caring for minority populations. The sites were sorted on the basis of geographic location, practice setting, and patient volume. Using retail prescription information, potential sites for inclusion into the registry, numbering 500, were identified, and the list was further modified to enrich representation of practices with intermediate patient volumes and of opinion leaders in the field. The final list of 500 sites had a geographic and physician specialty distribution similar to the original list of 4600 sites, and included 78 investigators considered key opinion leaders. Nephrologists had a $76 \%$ representation in the final 500 sites. The target enrollment was 120 sites, with a single principal investigator at each site, in order to achieve a final target of at least 100 sites actively recruiting patients over the course of the study $(20 \%$ projected site attrition). The target patient enrollment per site was 25 eligible patients within 3 months of site selection; some high-volume sites (academic centers) were permitted to exceed the target of 25 to achieve the expected target recruitment of 2000 participants.

\section{Patient Recruitment and Follow-Up}

In 1994 the National Institutes of Health (NIH) issued a consensus statement encouraging referral of patients to a nephrologist if serum creatinine was $\geq 1.5 \mathrm{mg} / \mathrm{dl}$ in females and $\geq 2 \mathrm{mg} / \mathrm{dl}$ in males. This has since been used as an operational definition of CKD (4). Inclusion and exclusion criteria for subjects were therefore broadly defined to include a representative mix of patients with recently diagnosed (incident) and established (prevalent) CKD. Prevalent subjects were defined as those under the care of the enrolling physician for longer than 6 months at the time of enrollment, and incident subjects were defined as those seen by the physician for less than 6 months prior to enrollment. Inclusion criteria were 18 years of age or older and GFR or creatinine clearance $(\mathrm{CrCl}) \leq 50 \%$ of expected value (approximately $60 \mathrm{ml} / \mathrm{min} / 1.73 \mathrm{~m}^{2}$ ) or a serum creatinine greater than $1.5 \mathrm{mg} / \mathrm{dl}$ for females or greater than $2 \mathrm{mg} / \mathrm{dl}$ for males. GFR was estimated using an equation derived from the Modification of Diet in Renal Disease (MDRD) study, which is based on age, gender, race, and serum creatinine $(13,16)$. Exclusion criteria were chronic dialysis at the time of enrollment, having received or been scheduled for a kidney transplant, anemia from causes other than kidney disease, or malignancy (other than basal cell) in the preceding 5 years.

Subjects were screened for eligibility by the treating physician during a routine clinic visit using a study eligibility criteria worksheet. Once a patient was defined as eligible and agreed to participate, informed consent was obtained by site personnel. Data were collected at enrollment and prospectively thereafter at follow-up visits. For prevalent patients, data were also collected retrospectively from the patient's chart from the time point of the first and midpoint visit to the enrolling physician. Follow-up visits and testing were as scheduled by the participating physician based on clinical need and not mandated by protocol. Follow-up was planned for 2 years or until the first of the following clinical outcomes: initiation of dialysis, death, or dropout from the study.

\section{Data Collection}

Data were collected by the enrolling physician on standardized patient status forms (PSFs) at enrollment and included baseline demographic, clinical, and laboratory data, past medical history, resource utilization, dietary recommendations, and prescribed medications. Demographic data included gender, race, date of birth, marital status, zip code, type of insurance, employment, and education. Clinical data included the primary cause of kidney disease, blood pressure, and current coexistent illnesses. Laboratory tests for assessment of kidney function and anemia, as well as certain other assays for liver function and diabetes control were collected. Medications of specific interest were erythropoietin (EPO), other antianemic agents, angiotensin-converting enzyme (ACE) inhibitors, and other antihypertensives. Resource utilization data included hospitalizations recommended by the physician, and patient reported hospitalizations and/or emergency room (ER) visits occurring between physician visits, procedures performed or ordered during the patient visit, referrals to other providers, and time to next follow-up. For the prevalent cohort, laboratory and clinical data were also collected retrospectively from the time of diagnosis of CKD or the first availability of recorded data, and at a midpoint between this and enrollment into the registry. At subsequent physician-directed follow-up visits, all of the information collected at baseline was updated whenever available. Insurance information, resource utilization, procedures ordered and/or performed, referrals to other providers, and planned time to next physician follow-up were recorded. 


\section{Health Related Quality of Life Assessment and Instruments}

A subset of 800 patients underwent a health-related quality of life (HRQoL) assessment obtained by telephone interview at enrollment and on a semiannual basis. The HRQoL assessment incorporated the Short Form 36 (SF-36) (17), the Kidney Disease Quality of Life (KD-QoL) (18), and the Functional Assessment of Cancer Therapy-Anemia (FACT-An) (19) instruments. The comprehensive assessment captured all eight SF-36 domain scores, including physical functioning, role-physical, bodily pain, general health, energy/vitality, social functioning, role-emotional and mental health, and two summary index scores for physical functioning and mental functioning. The KD-QoL domains used were those related to burden of disease, quality of social interaction, cognitive functioning, symptoms and effects of kidney disease, and sleep patterns. The entire FACTAn instrument was included and consisted of the general FACT questionnaire (FACT-G) that measures general quality of life as well as 20 additional items related to the impact of fatigue and anemia-related symptoms on quality of life. Also included were questions developed specifically for this study that address patient work and productivity levels, health care resource use, health and family disease history, and satisfaction with care.

\section{Statistical Analysis}

Cross-sectional analyses of baseline data at the time of enrollment include descriptive statistics such as mean, median, range and quartiles for continuous variables, and proportions for categorical data. Stratification by prevalent versus incident status, and selected demographic and clinical characteristics will be carried out to evaluate their relationship to laboratory data and future clinical outcomes. Of specific interest is describing the frequency of use of accepted interventions such as management of hypertension, diabetes, anemia, and the progression of CKD. The independent samples $t$-test and chi-squared test are applied to assess the significance of differences as appropriate for the data. Longitudinal analyses are carried out for the entire cohort and separately for the prevalent and incident cohorts. Time zero for longitudinal analysis of the prevalent group is the date of enrollment, and pre-enrollment data are used for adjustment in multivariate analyses. Longitudinal analyses include calculation of the rate of progression of GFR and factors associated with more rapid progression, and determination of the relationship between level of GFR and anemia, hyperparathyroidism, and HRQoL. In addition, utilization of health care resources, including hospitalization, emergency room visits, alternative health care, and other physician interventions are assessed. Factors associated with decreased HRQoL and increased morbidity and mortality are examined. Of specific interest is the examination of the impact of resource utilization and optimal anemia management on clinical outcomes. Linear and logistic regression analyses are used for continuous and categorical outcomes, respectively. Cox proportional hazards analyses or generalized estimating equation methods are used for time-to-event analyses or repeated outcomes analyses, respectively, with the adjustment of covariates in a timedependent fashion, if appropriate.

\section{Discussion}

To optimally address the health needs of the CKD population, attention will have to be shifted to earlier phases of the disease. The focus of much research thus far has been the care and outcomes of patients with ESRD. A better understanding of the current patterns of practice for diagnosis, evaluation, and management of CKD and its attendant comorbid conditions and their relationship to progression and other outcomes in CKD is required in order to improve outcomes among CKD patients. Epidemiologic data regarding CKD remains imprecise. This is likely related, in part, to difficulties with its early recognition, emphasizing the need for systematic and organized study in this area (14). To date, there has been no other large-scale, representative, prospective study that has addressed this issue. Outcomes research to assess the interrelationships among various treatment practices, patient demographics, complications, mortality, and other outcome risks in the CKD population has been long awaited. NIH has recently designated CKD as a priority area for study $(20,21)$, establishing the Chronic Renal Insufficiency Cohort study. This study, presently in its developmental stages, is expected to answer important questions, but data will not become available for several years. STRIDE, initiated in October 2000, has ongoing active recruitment and will soon have baseline and longitudinal information for analysis, and is expected to provide a valuable overview of CKD care in this country.

STRIDE has been designed to capture treatment patterns and to assess their relationship to patient outcomes in a nationally representative cohort of patients. There is particular emphasis on the impact of baseline kidney function and rates of disease progression, medications, comorbid conditions, referral patterns, and hospitalizations. This initial report focuses on the methods, design, and scope of the study, and demonstrates the feasibility of implementing such a registry. An observational study such as this is an efficient means of exploring various longitudinal and cross-sectional research questions, taking into account the heterogeneity inherent in population characteristics, existing practice patterns and organizational settings, and quality and standards of care (22). A similar study in the dialysis population has prompted changes in practice and formed the basis of clinical trials (23).

While there is a wealth of literature referring to HRQoL in the dialysis population, few studies have addressed this issue in CKD (24). This aspect of STRIDE is expected to be one of its major strengths; a very comprehensive instrument that will be employed on a large sample size and capture both cross-sectional and longitudinal trends. The study is expected to have sufficient power to relate HRQoL measures, including 
patient satisfaction with care, work, and productivity levels, to disease-specific parameters such as level of kidney function and anemia.

One of the problem areas in the study of CKD has been early diagnosis and recognition. While the onset of dialysis or transplantation is an easily identifiable final phase of CKD, the beginning is often not clearly defined or recognized, as many of the affected individuals are asymptomatic and thus may not even be under the care of a physician. The criteria for patient recruitment in this study may bias the cohort toward relatively more informed and motivated individuals. Consequently the sample may not be representative of the "universe" of patients with CKD, as it is only composed of those patients followed by physicians. This would be true of any such cohort study. The lack of physician visits or laboratory tests mandated by protocol is not necessarily a limitation in a prospective observational study, because the intent of this registry is to observe practice patterns rather than dictate them. Notwithstanding, the results of the STRIDE registry are expected to provide useful information to physicians caring for patients with $\mathrm{CKD}$, and for researchers to pursue further studies directed at improving the care of these patients, in particular, as it will analyze the impact of existing practices.

It appears clear from published literature that improvements in the care of patients with CKD before ESRD are necessary. However, knowledge regarding current practice patterns needs to be broadened so that changes in policy rest on sound information rather than the small observational studies published thus far $(7,9)$. The role of referral to a nephrologist earlier in the course of CKD (25), the role of mild kidney dysfunction as an important cardiovascular risk factor (26), and the benefit of optimal management of the complications associated with kidney disease need to be better defined in order to change existing practices. In fact, the U.S. Department of Health and Human Services' Healthy People 2010 objectives include a specific section regarding the impact of CKD and the need for improvements in the care of CKD patients, with a focus on better management of cardiovascular comorbidities (21). The STRIDE registry is expected to enhance the understanding of the impact of certain clinical practices on outcomes and thus provide sufficient rationale to promote improved practices.

\section{Acknowledgments}

The STRIDE registry is supported by Amgen Inc. The authors would like to acknowledge the members of the inaugural Registry Advisory Panel for their contribution to the development of the STRIDE registry: Nina Caplin, MD (Mt. Sinai Medical Center), John Brian Copley, MD (Oschner Medical Clinic), William Henrich, MD (University of Maryland Hospital), Jill Lindberg, MD (Oschner Medical Clinic), and Pedro Vergne-Marini, MD (Dallas Nephrology Associates).

\section{References}

1. U.S. Renal Data System: USRDS 2001 Annual Data Report. Bethesda, MD: National Institute of Health, National Institute of Diabetes and Digestive and Kidney Disease, 2001

2. U.S. Renal Data System: USRDS 2000 Annual Data Report. Bethesda, MD: National Institute of Health, National Institute of Diabetes and Digestive and Kidney Disease, 2000

3. NIH Consensus Statement: Morbidity and mortality of dialysis. Ann Intern Med 121:62-70, 1994

4. Obrador GT, Ruthazer R, Arora P, Kausz AT, Pereira BJG: Prevalence of and factors associated with suboptimal care before initiation of dialysis in the United States. J Am Soc Nephrol 10:1793-1800, 1999

5. U.S. Renal Data System: USRDS 1997 Annual Data Report. Bethesda, MD: National Institute of Health, National Institute of Diabetes and Digestive and Kidney Disease, 1997

6. Van Biesen W, Wiedemann M, Lamiere N: End-stage renal disease treatment: a European perspective. J Am Soc Nephrol 9(suppl):S55-S62, 1998

7. Arora P, Obrador GT, Ruthazer R, Kausz AT, Meyer KB, Jenuleson CS, Pereira BJG: Prevalence, predictors and consequences of late nephrology referral at a tertiary care center. J Am Soc Nephrol 6:1281-1286, 1999

8. Levin A, Lewis M, Mortiboy P, Faber S, Hare I, Porter EC, Mendelssohn DC: Multidisciplinary predialysis programs: quantification and limitations of their impact on patient outcomes in two Canadian settings. Am J Kidney Dis 29:533-540, 1997

9. Obrador GT, Pereira BJG: Early referral to the nephrologist and timely initiation of renal replacement therapy - a paradigm shift in the management of patients with chronic renal failure. Am J Kidney Dis 31:398-417, 1998

10. Kausz AT, Khan SS, Abichandani R, Kazmi WH, Obrador GT, Ruthazer R, Pereira BJ: Management of patients with chronic renal insufficiency in the northeastern United States. J Am Soc Nephrol 12:1501-1507, 2001

11. Jones C, McQuillan GM, Kusek JW, Eberhardt MS, Herman WH, Coresh J, Salive M, Jones CP, Agodoa LY: Serum creatinine levels in the US population: Third National Health and Nutrition Examination Survey. Am J Kidney Dis 32:992-999, 1998 (erratum: Am J Kidney Dis 35:178, 2000)

12. Nissenson A, Pereira BJG, Collins AJ, Steinberg EP: Prevalence and characteristics of individuals with chronic kidney disease in a large health maintenance organization. Am J Kidney Dis 37:1177-1183, 2001

13. National Kidney Foundation: K/DOQI clinical practice guidelines for chronic kidney disease: evaluation, classification, and stratification. Am J Kidney Dis 39(suppl 1):S1-S266, 2002

14. Bolton WK, Kliger AS: Chronic renal insufficiency: current understandings and their implications. Am J Kidney Dis 36(suppl 3):S4-S12, 2000

15. U.S. Food and Drug Administration: Good Clinical Practice in FDA Regulated Clinical Trials. Washington, DC: U.S. Food and Drug Administration, 2001

16. Levey A, Bosch JP, Lewis JB, Greene T, Rogers N, Roth D: A more accurate method to estimate glomerular filtration rate from serum creatinine: a new prediction equation. Ann Intern Med 130:461-470, 1999

17. McHorney CA, Ware JE, Lu JFR, Sherbourne CD: The MOS 36-item shortform health survey (SF-36): III. tests of data quality, scaling assumptions and reliability across diverse patient groups. Med Care 32:40-66, 1994

18. Hays RD, Kallich JD, Mapes DL, Coons SJ, Carter WB: Development of the kidney disease quality of life (KDQOL) instrument. Qual Life Res 3:329-338, 1994

19. Cella D: The Functional Assessment of Cancer Therapy-Anemia (FACTAn) Scale: a new tool for the assessment of outcomes in cancer anemia and fatigue. Semin Hematol 34:13-19, 1997

20. Request for applications: DK-01-005 National Institute of Diabetes and Digestive and Kidney Diseases. Prospective cohort study of chronic renal insufficiency (CRIC study grant summaries), 2000 URL: http:/www. niddk.nih.gov/patient/cric/cric.htm

21. U.S. Department of Health and Human Services: Healthy People 2010, 2nd ed. Washington, DC: U.S. Government Printing Office, 2000 URL: http:// www.health.gov/healthypeople/

22. Zoccali C: The cost of knowledge in nephrology and the importance of clinical databases. $J$ Nephrol 13:43-45, 2000

23. Young EW, Goodkin DA, Mapes DL, Port FK, Keen ML, Chen K, Maroni BL, Wolfe RA, Held PJ: The Dialysis Outcomes and Practice Patterns Study (DOPPS): an international hemodialysis study. Kidney Int 57(suppl 74):S74 $\mathrm{S} 81,2000$

24. Valderrabano F, Jofre R, Lopez-Gomez JM: Quality of life in end stage renal disease patients. Am J Kidney Dis 38:443-464, 2001

25. Nissenson AR, Collins AJ, Hurley J, Petersen H, Pereira BJG, Steinberg EP. Opportunities for improving the care of patients with chronic renal insufficiency: current practice patterns. J Am Soc Nephrol 12:1713-1720, 2001

26. Mann JF, Gerstein HC, Pogue J, Bosch J, Yusuf S: Renal insufficiency as a predictor of cardiovascular outcomes and the impact of ramipril: the HOPE randomized trial. Ann Intern Med 134:629-636, 2001 